\title{
Ocular manifestations of graft-versus-host disease: 10 years' experience
}

This article was published in the following Dove Press journal:

Clinical Ophthalmology

3 July 2015

Number of times this article has been viewed

\author{
Xihui Lin \\ Harrison Dwight Cavanagh
}

Department of Ophthalmology, University of Texas Southwestern Medical Center, Dallas, TX, USA
Correspondence: Harrison Dwight

Cavanagh

Department of Ophthalmology,

University of Texas Southwestern

Medical Center, 5323 Harry Hines

Boulevard, Dallas, TX 75390-9057, USA

Email dwight.cavanagh@utsouthwestern. edu
Purpose: To evaluate the ocular presentation, treatment, and clinical course of graft-versus-host disease (GVHD).

Design: Retrospective case series.

Participants: Two hundred and forty-nine patients with systemic GVHD were included in the study.

Methods: Ocular and systemic data were collected from 2003 to 2013.

Main outcome measures: Mortality, visual acuity, and response of ocular symptoms.

Results: Sixty-four patients had ocular manifestations (25.7\%). At presentation, the mean age was 44.5 years and mean latency was 16.4 months. The most common presentations were keratoconjunctivitis sicca, cataract, blepharitis, ocular hypertension, and filamentary keratitis. Visual acuity at presentation was 20/49; at the worst point in the disease was 20/115; and at most recent visit was $20 / 63$. When topical anti-inflammatory drops were used in addition to tears, $54.3 \%$ of patients' ocular symptoms stabilized. When autologous serum was used in addition, $80 \%$ stabilized. The overall 10 -year mortality of GVHD was $29.7 \%$. For those with ocular involvement, it was $21.9 \%$.

Conclusion: Systemic GVHD has a high mortality rate, but ocular involvement does not suggest a worse prognosis. The main ocular presentations were keratoconjunctivitis sicca, cataracts, and ocular hypertension. Dry eyes in this population were very severe with overall worsening in visual acuity. However, with a step-wise approach involving topical anti-inflammatory medications and autologous serum tears, ocular symptoms do improve. It is important to monitor these patients closely, as they are prone to serious ocular complications such as corneal perforation and endophthalmitis.

Keywords: dry eye, keratitis, corneal ulceration

\section{Introduction}

Allogeneic stem cell transplantation has become the standard of care for a variety of hematologic malignancies as well as hematopoietic aplasia and dysplasia. ${ }^{1}$ Part of the success of the therapy relies on the graft-versus-tumor effect, but, as a consequence, a significant number of recipients develop graft-versus-host disease (GVHD) due to the host-targeted alloreactive response. ${ }^{2}$ The incidence of GVHD reported in 1974 was $70.5 \% .^{3}$ Even with newer immunosuppressive medications and methodologies, the incidence is still between $25 \%$ and $70 \%$ today.

GVHD can be divided into acute and chronic stages depending on whether symptoms occur before or after 100 days post-engraftment. ${ }^{5}$ Any system can be affected, but the most common include those of skin, gastrointestinal system, liver, and eyes. ${ }^{6}$ Ocular manifestations affect a significant number of patients after allogeneic stem cell transplant and have been reported to be as high as $60 \%$ in patients with systemic 
GVHD. ${ }^{7}$ Classically, ocular GVHD has been defined by keratoconjunctivitis sicca (KCS) and ocular surface inflammation following stem cell transplantation. ${ }^{8}$ The pathophysiological response involves primarily $\mathrm{T}$-cell-mediated targeting against recipient tissues involving IL-1, IL-2, interferon-gamma, tumor necrosis factor-alpha, and other cytokines. ${ }^{9}$ In the eye, these responses are principally directed against the conjunctiva, lacrimal gland, and accessory tear glands. ${ }^{10}$

The sporadic GVHD literature on ocular disease manifestations of GVHD and treatment modalities has been limited by the lack of long-term follow-up reporting on mortality and visual prognosis. In this study, we report our 10-year experience with ocular GVHD presentations, treatment modalities, and clinical outcomes.

\section{Methods}

Institutional review board approval was obtained and a retrospective chart search was conducted to identify all patients from January 2003 to October 2013 with a diagnosis of GVHD seen at University of Texas Southwestern Medical Center, Parkland Memorial Hospital, or the Children's Medical Center of Dallas (Dallas, TX, USA). Patients presenting to the eye clinic with first-time ophthalmic complaints and patients with new eye complaints managed by their primary care provider or oncologist were included in the study. Systemic GVHD was defined by immune-mediated hepatitis, skin rash, diarrhea, pneumonitis, or hematopoietic involvement in the setting of post-stem cell transplantation. Classic ocular GVHD was defined by those with: 1) previous stem cell transplantation; 2) new onset of dry eye symptoms such as dryness, burning, or foreign-body sensation; and 3) objective findings of KCS such as short tear break-up time, decreased Schirmer's test, or uptake of corneal vital staining. Patients with other ocular manifestations were also analyzed. Patients with preexisting eye problems without new signs or symptoms after the transplant were excluded.

Institutional review board approval was also received for a standardized protocol for preparation, storage, and use of the patient's own autologous serum at a non-diluted concentration. Autologous serum tears were prepared by spinning down a patient's whole blood then collecting the serum into individual dropper bottles without dilution. The unopened bottles were stored at $-20^{\circ} \mathrm{C}$, and the opened bottle was refrigerated at $4^{\circ} \mathrm{C}$ until application.

Of patients with available data, the age at presentation, sex, ethnicity, and latent period from infusion of stem cells to ocular symptoms were identified. The presenting visual acuity in the more affected eye, the worst visual acuity during the follow-up period, and the visual acuity at the most recent follow-up were recorded. In addition, the presenting ocular signs and symptoms, treatments used, response to treatment, follow-up duration, and systemic steroid status were noted. In addition, mortality rates were analyzed. Statistical analysis of the responses to treatment and mortality data were performed using a chi-square test while quantitative data such as visual acuity comparisons were done with Student's $t$-test with significance set at $P<0.05$.

\section{Results}

Of the 249 patients with GVHD reviewed, 64 met the inclusion criteria of having new ocular manifestations (25.7\%). Seven of the 64 patients were less than 18 years old (10.9\%). Patient demographics in ocular GVHD are shown in Table 1. The average age at presentation was 44.5 years, and the average latency from transplant to presentation was 16.4 months.

Table 2 shows visual acuity at different time points: at presentation, the average visual acuity in the more affected eye was 20/49 (logMAR 0.386). Of the patients seen more than once, the average visual acuity at the worst point in the disease was 20/115 (logMAR 0.758). The average visual acuity at the most recent visit was 20/63 (logMAR 0.502).

Table 3 reports all ocular manifestations: 49 of 61 had classic ocular GVHD (KCS, 80.3\%). Of the other ocular manifestations, 23 out of 61 had visually significant cataract requiring surgery (37.7\%); eleven of 61 had blepharitis (18.0\%); nine of 61 had ocular hypertension (14.8\%); and six of 61 had filamentary keratitis $(9.8 \%)$. Of the patients with ocular hypertension, two were started on glaucoma medications. Three patients had corneal perforations due to infectious keratitis, of which one had separate perforations in each eye. One patient had endogenous Fusarium endophthalmitis and one had a new choroidal lymphoma with serous retinal detachment. The triad of KCS, cataract, and ocular hypertension was the most common ocular presentations. Fifty-seven of 61 patients $(93.4 \%)$ had one or more of these three.

Of the 49 patients presenting with classic ocular GVHD, only four were successfully treated with artificial tears alone

Table I Patient demographics

\begin{tabular}{ll}
\hline & $\mathbf{n ~ ( \% )}$ \\
\hline Male & $37(57.8 \%)$ \\
Female & $27(42.2 \%)$ \\
Caucasian & $46(71.8 \%)$ \\
Hispanic & $9(14.1 \%)$ \\
African-American & $5(7.8 \%)$ \\
Asian & $2(3.1 \%)$ \\
Other & $2(3.1 \%)$ \\
\hline
\end{tabular}


Table 2 Visual acuity

\begin{tabular}{lll}
\hline Time point & logMAR acuity & Snellen acuity \\
\hline Initial presentation & 0.386 & $20 / 49$ \\
Worst point in disease & 0.758 & $20 / 115$ \\
Most recent visit & 0.502 & $20 / 63$ \\
\hline
\end{tabular}

(8.2\%). Twenty-six required topical cyclosporine (53.1\%); 18 required topical steroids (prednisolone, fluorometholone, or loteprednol; 36.7\%); 15 required autologous serum tears in $100 \%$ concentration (30.6\%); ten required bandage contact lenses (20.4\%); eight underwent punctal occlusion (16.3\%); and three required penetrating keratoplasty for complications related to KCS (Table 4). For patients whose artificial tear usage frequencies were available, 15 out of 37 used them at least once per hour (40.5\%). Compared to artificial tears alone, when patients were on topical anti-inflammatory drops (cyclosporine, steroids, or both), 19 of 35 had improvement of symptoms $(54.3 \%, P<0.001)$. When autologous serum was added, eight of ten had improvement of symptoms and required no other treatments $(80.0 \%, P<0.001)$. When autologous serum was used alone without topical steroids or cyclosporine, three of five required no other treatments $(60 \%, P=0.013)$. For patients treated with topical anti-inflammatory medications, the initial and final average visual acuities were 20/46 and $20 / 74$, respectively $(P=0.12)$. For those treated with autologous serum, the initial and final average visual acuities were $20 / 77$ and 20/74, respectively ( $P=0.93$ ). There were no adverse events related to the usage of autologous serum tears.

All patients were on tacrolimus or mycophenolate mofetil for prophylaxis of GVHD. The prophylaxis regimen did not appear to correlate with the incidence or severity of ocular GVHD. At the most recent follow-up, 42 of 57 patients with systemic medication lists remained on oral steroids $(73.7 \%)$.

Table 3 Ocular manifestations

\begin{tabular}{ll}
\hline & $\mathbf{n}(\%)$ \\
\hline Classic ocular GVHD (KCS) & $49(80.3 \%)$ \\
Visually significant cataract & $23(37.7 \%)$ \\
Blepharitis & $\mathrm{I}$ I (I8.0\%) \\
Ocular hypertension & $9(14.8 \%)$ \\
Filamentary keratitis & $6(9.8 \%)$ \\
Corneal perforation & $3(4.9 \%)$ \\
Herpetic keratitis & $2(3.3 \%)$ \\
Bacterial/fungal corneal ulcer & $2(3.3 \%)$ \\
Optic neuritis/neuropathy & $2(3.3 \%)$ \\
Bilateral anterior uveitis & $\mathrm{I}(\mathrm{I} .6 \%)$ \\
Endophthalmitis & $\mathrm{I}(\mathrm{I} .6 \%)$ \\
Serous retinal detachment with choroidal lymphoma & $\mathrm{I}(\mathrm{I} .6 \%)$ \\
Punctal stenosis & $\mathrm{I}(\mathrm{I} .6 \%)$ \\
\hline
\end{tabular}

Abbreviations: GVHD, graft-versus-host disease; KCS, keratoconjunctivitis sicca.
Table 4 Treatments for keratoconjunctivitis sicca

\begin{tabular}{ll}
\hline & $\mathbf{n ~ ( \% )}$ \\
\hline Artificial tears & $49(100 \%)$ \\
Topical cyclosporine and/or steroids & $35(71.4 \%)$ \\
Topical cyclosporine & $26(53.1 \%)$ \\
Topical steroids & $18(36.7 \%)$ \\
Autologous serum tears (I00\%) & $15(30.6 \%)$ \\
Bandage contact lens & $10(20.4 \%)$ \\
Punctal occlusion & $8(16.3 \%)$ \\
Penetrating keratoplasty & $3(6.1 \%)$ \\
\hline
\end{tabular}

For patients presenting with systemic GVHD, the mortality rate during this 10 -year period was $29.7 \%$ (74 of 249 ); for those with ocular GVHD, the mortality rate was $21.9 \%$ (14 of 64). Presenting patients without ocular involvement had a mortality rate of $32.4 \%$ ( 60 of $185, P=0.15$ ). Patients with classic ocular GVHD had a mortality rate of $22.4 \%$ (eleven of 49), while the remainder had a mortality rate of $20.0 \%$ (three of $15, P=0.84$ ).

\section{Discussion}

The $25.7 \%$ incidence of ocular involvement in this study is lower than that of previous prevalence studies, in which the incidence was closer to $50 \% .^{11,12}$ This decrease may be explained by the difference in the referral protocol between institutions: many patients with mild ocular symptoms were managed by the oncologist without ophthalmology referral until artificial tear usage became too frequent or vision worsened. The time frames between the two cohorts differed as well. A previous study reported outcomes between 1990 and 2006 and the current study focused on 2003-2013. ${ }^{12}$ Differences in surveillance methods and improved management protocols of GVHD also likely accounted for some of the difference. The average time until presentation of 16.4 months was within the range reported by previous literature (9.8-32 months). ${ }^{13,15}$

Previous studies have defined ocular GVHD as new or worsening KCS occurring post-stem cell engraftment. ${ }^{8,11,14,15}$ While the majority of patients in this study fit the criteria for classic ocular GVHD, 70\% also had other ocular manifestations. Cataracts and ocular hypertension were among the most common. Part of the etiology is likely due to systemic and topical steroid usage, as almost all patients with GVHD at some point had prolonged use of systemic steroids and more than $70 \%$ were still receiving them at the most recent follow-up. In addition, the primary inflammatory response related to the GVHD itself may contribute to the pathogenesis. Blepharitis and filamentary keratitis are likely secondary to the inflammatory ocular surface disease. 
The KCS in the reported population appears to be more severe compared to previous studies. ${ }^{10-12,14}$ At our institution, the typical management of KCS when tears alone are not sufficient is the addition of topical anti-inflammatory medications such as cyclosporine, loteprednol, or prednisolone acetate. When the disease is still not controlled, the next step is practitioner dependent. Many physicians at our institution have found success with $100 \%$ autologous serum tears. In this study, more than $90 \%$ of the patients required one or more therapies in addition to artificial tears. When topical antiinflammatory therapy was added, $54.3 \%$ had improvement of symptoms. Of the remaining, when non-diluted autologous serum tears were added, $80 \%$ had improvement and required no other treatments. Although not statistically significant, patients treated with topical anti-inflammatory drops trended toward visual acuity deterioration (20/46 to $20 / 74, P=0.12$ ), while those treated with autologous serum seemed stabilized (20/77 to $20 / 74, P=0.93$ ); however, patients who were started on autologous serum usually presented with more advanced initial disease. On average, the ocular surface disease appeared progressive, with vision worsening from 20/50 to $20 / 115$, but, with aggressive treatment, improved to 20/63 at the most recent visit. A subset of patients (eleven of 41 , $26.7 \%$ ), despite aggressive management of the ocular surface disease, deteriorated to a visual acuity worse than 20/100 at the most recent follow-up. Two of these cases, however, were complicated by posterior segmented pathology: one had fungal endophthalmitis and another had a recurrent choroidal lymphoma. Cataract surgery likely contributed to some of the improvement in final visual acuity compared to the worst visual acuity. However, cataract occurrence appeared to be similar in the KCS treatment subgroups and therefore was not likely to have contributed significantly to the differences among those groups.

Corneal ulceration leading to perforations in ocular GVHD was not uncommon. ${ }^{16}$ All three of our cases requiring penetrating keratoplasty were due to an infectious ulcer with progressive thinning, and eventually perforated. Application of bandage contacts and tissue adhesive glue were attempted in two of the cases with no improvement. Bacterial keratitis was common in this population due to poor surface integrity and intense immunosuppression. The rapid progression of infectious ulcers was likely due to a combination of poor wound healing from severe dryness, chronic steroid usage, and an altered immune response from systemic immune suppression and GVHD itself.

The incidence of herpetic eye disease at 3.3\% was not significantly different than that of the general population. ${ }^{17}$
We expected the rate to be higher given the increased rate of immune suppression and topical steroids in this group.

Some of the ocular manifestations observed may not result directly from GVHD. One patient developed endogenous Fusarium endophthalmitis likely secondary to the altered immune function in a post-marrow transplant state and immunosuppressive medications. One had serous retinal detachment with choroidal lymphoma likely due to a recurrence of the initial malignancy. Spontaneous punctal occlusions, however, have been reported to occur more frequently in chronic GVHD patients. ${ }^{18}$

The usage of autologous serum in this group of patients is somewhat paradoxical due to the concern of worsening alloreactive response when serum containing anti-host antibodies generated by the graft immune system is exposed to host ocular surface antigens. However, several other studies have found similar benefits with autologous serum..$^{19,20}$ This may be due to immune tolerance induced by the differences in immunoglobulin subtypes in serum versus the mucosal surface. Further studies are warranted to elucidate specific mechanisms. Another recent approach for severe KCS is the usage of scleral contact lenses with prosthetic replacement of the ocular surface (PROSE). Our study included one patient treated by such an approach with improvement and stabilization of his vision and symptoms over a 2-year period. At our institution, the algorithm for GVHD starts with frequent preservative-free artificial tears with close monitoring. If this is not sufficient, topical anti-inflammatory drops such as cyclosporine and/or steroids are added. If symptoms worsen despite these, $100 \%$ autologous serum tears are used in addition. The next step in the algorithm may require referral to a center specializing in the PROSE system.

Overall, patients presenting with systemic GVHD had a nearly $30 \%$ all-cause mortality rate over the 10 -year study period. The difference in mortality rate of those presenting with ocular manifestations compared with those without was not statistically significant $(P=0.15)$, but there was a trend toward a lower mortality rate in those with ocular involvement. This was not expected but can likely be explained if many mortalities occur during the latent period between engraftment and the onset of ocular symptoms. Specific ocular GVHD presentations did not correlate with mortality.

In summary, the majority of ocular GVHD cases presented with symptomatic KCS, but also had other ocular manifestations including cataract, ocular hypertension, and filamentary keratitis. The KCS in this population can be very severe and progressive despite aggressive lubrication. Topical anti-inflammatory therapy and autologous serum offer 
significant improvement over tears alone. However, despite these interventions, a small group continues to worsen and may develop progressive nonhealing corneal ulcerations eventually necessitating keratoplasty procedures. When faced with patients referred for GVHD, it is important to recognize ocular manifestations and initiate early aggressive treatment of any ocular surface disease. Because systemic and/or topical use of corticosteroids may be necessary for indefinite periods, it is also important to follow closely intraocular pressures and intervene medically and/or surgically as needed.

\section{Acknowledgment}

This study was supported in part by an unrestricted grant from Research to Prevent Blindness, New York, NY, USA; and by EY-R020799.

\section{Disclosure}

The authors report no conflicts of interest in this work.

\section{References}

1. Malatack JJ, Consolini DM, Bayever E. The status of hematopoietic stem cell transplantation in lysosomal storage disease. Pediatr Neurol. 2003;29:391-403.

2. Petersen SL. Alloreactivity as therapeutic principle in the treatment of hematologic malignancies. Studies of clinical and immunologic aspects of allogeneic hematopoietic cell transplantation with nonmyeloablative conditioning. Dan Med Bull. 2007;54(2):112-139.

3. Glucksberg H, Storb R, Fefer A, et al. Clinical manifestations of graftversus-host disease in human recipients of marrow from HL-A-matched sibling donors. Transplantation. 1974;18:295-304.

4. Dean RM, Bishop MR. Graft-versus-host disease: emerging concepts in prevention and therapy. Curr Hematol Rep. 2003;2:287-294.

5. Filipovich AH, Weisdorf D, Pavletic S, et al. National Institutes of Health consensus development project on criteria for clinical trials in chronic graft-versus-host disease: I. Diagnosis and staging working group report. Biol Blood Marrow Transplant. 2005;11:945-956.
6. Ferrara JL, Levine JE, Reddy P, Holler E. Graft-versus-host disease. Lancet. 2009;373:1550-1561.

7. Balaram M, Dana MR. Phacoemulsification in patients after allogeneic bone marrow transplantation. Ophthalmology. 2001;108:1682-1687.

8. Franklin RM, Kenyon KR, Tutschka PJ, Saral R, Green WR, Santos GW Ocular manifestations of graft-vs-host disease. Ophthalmology. 1983; 90:4-13.

9. Ferrara JL, Reddy P. Pathophysiology of graft-versus-host disease. Semin Hematol. 2006;43:3-10.

10. Saito T, Shinagawa K, Takenaka K, et al. Ocular manifestation of acute graft-versus-host disease after allogeneic peripheral blood stem cell transplantation. Int J Hematol. 2002;75:332-334.

11. Westeneng AC, Hettinga Y, Lokhorst H, Verdonck L, van Dorp S, Rothova A. Ocular graft-versus-host disease after allogeneic stem cell transplantation. Cornea. 2010;29(7):758-763.

12. Allan EJ, Flowers ME, Lin MP, Bensinger RE, Martin PJ, Wu MC. Visual acuity and anterior segment findings in chronic graft-versus-host disease. Cornea. 2011;30(12):1392-1397.

13. Vanathi M, Kashyap S, Khan R, et al. Ocular surface evaluation in allogenic hematopoietic stem cell transplantation patients. Eur $J$ Ophthalmol. 2014;24(5):655-666.

14. Nassiri N, Eslani M, Panahi N, Mehravaran S, Ziaei A, Djalilian AR. Ocular graft versus host disease following allogeneic stem cell transplantation: a review of current knowledge and recommendations. J Ophthalmic Vis Res. 2013;8(4):351-358.

15. Shikari H, Amparo F, Saboo U, Dana R. Onset of ocular graft-versushost disease symptoms after allogeneic hematopoietic stem cell transplantation. Cornea. 2015;34:243-247.

16. Stevenson W, Shikari H, Saboo US, Amparo F, Dana R. Bilateral corneal ulceration in ocular graft-versus-host disease. Clin Ophthalmol. 2013; 7:2153-2158.

17. Uchio E, Hatano K, Mitsui K, et al. A retrospective study of herpes simplex keratitis over the last 30 years. Jpn J Ophthalmol. 1994; 38:196-201.

18. Kamoi M, Ogawa Y, Dogru M, et al. Spontaneous lacrimal punctal occlusion associated with ocular chronic graft-versus-host disease. Curr Eye Res. 2007;32(10):837-842.

19. Ogawa Y, Okamoto S, Mori T, et al. Autologous serum eye drops for the treatment of severe dry eye in patients with chronic graft-versus-host disease. Bone Marrow Transplant. 2003;31:579-583.

20. Rocha EM, Pelegrino FSA, de Paiva CS, Vigorito AC, de Souza CA. GVHD dry eyes treated with autologous serum tears. Bone Marrow Transplant. 2000;25:1101-1103.
Clinical Ophthalmology

\section{Publish your work in this journal}

Clinical Ophthalmology is an international, peer-reviewed journa covering all subspecialties within ophthalmology. Key topics include: Optometry; Visual science; Pharmacology and drug therapy in eye diseases; Basic Sciences; Primary and Secondary eye care; Patient Safety and Quality of Care Improvements. This journal is indexed on

\section{Dovepress}

PubMed Central and CAS, and is the official journal of The Society of Clinical Ophthalmology (SCO). The manuscript management system is completely online and includes a very quick and fair peer-review system, which is all easy to use. Visit http://www.dovepress.com/ testimonials.php to read real quotes from published authors. 